\title{
The diagnostic accuracy of integrated positron emission tomography/computed tomography in the evaluation of pulmonary mass lesions in a tuberculosis-endemic area
}

\author{
R du Toit, ${ }^{1}$ MB ChB, FCP (SA), MMed (Int); J A Shaw, ${ }^{1}$ MB ChB; E M Irusen, ${ }^{1}$ MB ChB, FCP (SA), FCCP, PhD; \\ F von Groote-Bidlingmaier, ${ }^{1} \mathrm{MD} ; \mathbf{J}$ M Warwick, ${ }^{2} \mathrm{MB} \mathrm{ChB}, \mathrm{FCNP}(\mathrm{SA}), \mathrm{PhD}$; \\ C F N Koegelenberg, ${ }^{1} \mathrm{MB}$ ChB, MMed (Int), FCP (SA), FRCP (UK), Cert Pulm (SA), PhD
${ }^{1}$ Division of Pulmonology, Department of Medicine, Faculty of Medicine and Health Sciences, Stellenbosch University and Tygerberg Academic Hospital, Cape Town, South Africa
${ }^{2}$ Division of Nuclear Medicine, Department of Medical Imaging and Clinical Oncology, Faculty of Medicine and Health Sciences, \\ Stellenbosch University and Tygerberg Academic Hospital, Cape Town, South Africa
}

Corresponding author: C F N Koegelenberg(coeniefn@sun.ac.za)

\begin{abstract}
Background. Integrated positron emission tomography/computed tomography (PET-CT) is a well-validated modality for assessing pulmonary mass lesions and specifically for estimating risk of malignancy. Tuberculosis (TB) is known to cause false-positive PET-CT findings.

Objective. To investigate the utility of PET-CT in the evaluation of pulmonary mass lesions and nodules in a high TB prevalence setting. Methods. All patients referred for the evaluation of a solitary pulmonary nodule or mass and who underwent PET-CT scanning over a 3 -year period were included. The PET-CT findings, including maximum standardised uptake value $\left(\mathrm{SUV}_{\max }\right)$, were compared with the gold standard (tissue or microbiological diagnosis). The sensitivity, specificity, positive and negative predictive values and diagnostic accuracy for malignant disease were calculated according to the $\mathrm{SUV}_{\max }$ cut-off of 2.5 and a proposed cut-off obtained from a receiver operating characteristic (ROC) curve.

Results. Forty-nine patients (mean (standard deviation) age 60.1 (10.2) years; 29 males) were included, of whom 30 had malignancy. Using an $\mathrm{SUV}_{\max }$ cut-off of 2.5, PET-CT had a sensitivity, specificity, positive and negative predictive value and diagnostic accuracy for

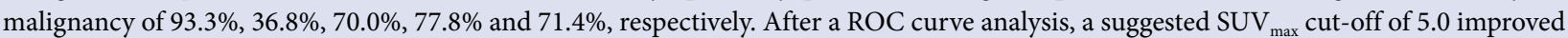
the specificity to $78.9 \%$ and the diagnostic accuracy to $86.7 \%$, with a small reduction in sensitivity to $90.0 \%$.

Conclusions. The diagnostic accuracy of PET-CT in the evaluation of pulmonary mass lesions using the conventional SUV max $_{\text {cut-off }}$ of 2.5 was reduced in a TB-endemic area. $\mathrm{An} \mathrm{SUV}_{\max }$ cut-off of 5.0 has a higher specificity and diagnostic accuracy for malignancy, with a comparable sensitivity.
\end{abstract}

S Afr Med J 2015;105(12):1049-1052. DOI:10.7196/SAMJ.2015.v105i12.10300

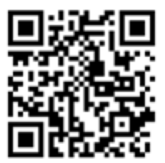

A solitary pulmonary nodule (SPN) or mass may either represent lung cancer or a benign disease such as pulmonary tuberculosis (TB). Most such lesions are incidental findings on a chest radiograph, and malignancy is generally actively excluded as it may represent curable disease. ${ }^{[1]}$ As patients with metastatic disease usually do not qualify for resection, the correct assessment and provisional staging of lung cancer patients is crucial in guiding their further management. Integrated positron emission tomography/computed tomography (PET-CT) is an integral part of this diagnostic process, and has been shown to reduce the number of futile thoracotomies. ${ }^{[2]}$

The majority of studies on PET-CT have been conducted in areas with a low or moderate prevalence of $\mathrm{TB}$, a disease which is well known to cause false-positive results. ${ }^{[3,4]}$ Developing regions, particularly TB-endemic areas, therefore face a potential challenge of interpreting PET-CT images as false positive. ${ }^{[5,6]}$

The aim of this study was to assess the sensitivity, specificity, and diagnostic accuracy of integrated PET-CT in the evaluation of SPNs and masses in an area with a very high TB incidence. A secondary aim was to investigate which maximum standardised uptake value $\left(\mathrm{SUV}_{\max }\right)$ cut-off would potentially be the most suitable in distinguishing between benign and malignant disease in a TB-endemic area. The overall objective of the study was to improve the management of pulmonary masses by preventing unnecessary thoracotomies for benign lesions.

\section{Methods \\ Study population}

All patients referred to the Division of Pulmonology at Tygerberg Academic Hospital, Cape Town, South Africa, from 2009 to 2012 for evaluation of apparent SPNs or masses $(1-6 \mathrm{~cm}$ in diameter) seen on routine chest radiographs and who subsequently underwent an integrated PET-CT were included. Patients who were found to have mediastinal lymph node involvement (not seen on routine chest radiography) on subsequent imaging (CT or PET-CT) were not excluded. Tygerberg Academic Hospital, a 1 380-bed academic hospital, is one of two academic referral centres in the city and renders a tertiary service to a population of approximately 1.5 million. According to the 2014 World Health Organization report, this population has one of the highest incidence rates for $\mathrm{TB}$, approximately 1000 cases for every 100000 people each year, and the third highest burden of disease globally after India and China. ${ }^{[7]}$

The study was approved by the Stellenbosch University Health Research Ethics Committee, and informed consent was obtained from all subjects prior to enrolment and prior to all invasive procedures. 


\section{Imaging techniques}

All studies were performed from the base of the skull to the upper thigh with a Siemens Biograph or Philips Gemini PET-CT with 16-slice uncontrasted CT. The procedure was performed according to European Association of Nuclear Medicine guidelines. ${ }^{[8]}$ Patients fasted, except for water intake, for at least 6 hours prior to the study. Blood glucose levels were tested prior to the scan, which was postponed if the patient had a level of $>11.1 \mathrm{mmol} / \mathrm{L}$. Patients lay supine for 30 minutes prior to the study, and for a further 60 minutes without talking after injection of ${ }^{18} \mathrm{~F}$-fluorodeoxyglucose (FDG). An FDG dose of 7 - $13 \mathrm{mCi}$ was administered according to body weight. All images were interpreted by an experienced nuclear medicine physician, at least one radiologist, and independently by a respiratory physician. Positivity was defined clinically as any FDG uptake greater than the mediastinal blood pool. The intensity of the FDG uptake in an area of interest was quantified using a Philips EBW workstation. A cursor was manually placed at the site of highest visual uptake within the lymph node and the $\mathrm{SUV}_{\max }$ was obtained from this slice using a circular region of interest with an $8 \mathrm{~mm}$ diameter.

\section{Tissue sampling}

The final diagnosis was established by histological or cytological analysis of tissue samples acquired from the pulmonary mass lesion or mediastinal lymph nodes, depending on the results of the PETCT. Patients with a positive result for the pulmonary mass lesion on integrated PET$\mathrm{CT}$ and negative results for mediastinal lymph node $\left(\mathrm{N}_{0}\right)$ and distant metastases $\left(\mathrm{M}_{0}\right)$ then underwent tissue sampling procedures of the pulmonary mass lesion. This was performed using CT-guided fineneedle aspiration, transthoracic ultrasoundguided needle aspiration or excision biopsy/ lobar resection as indicated. Patients with a negative integrated PET-CT result for distant metastases and a positive result for mediastinal lymph node involvement (positive $\mathrm{N}$-staging) underwent either endoscopic sampling of the mediastinal lymph nodes or tissue acquisition by mediastinoscopy, depending on their accessibility.

Because of the high negative predictive value of integrated PET-CT, negative $\mathrm{N}$-staging and $\mathrm{M}$-staging on PET-CT was considered to be a true negative. This was then confirmed with clinical and radiological follow-up. Each patient included in the study was also tested for Mycobacterium tuberculosis infection. This was done by Ziehl-Nielsen staining and culture of sputum and/or bronchial washings, and/or histological proof of granulomatous tissue with necrosis, and/or culture of fine-needle aspirates.

Benign disease was defined as TB proven by the methods outlined above, histological confirmation of benign disease on lung resection, or clinical and radiological followup for a period of at least 2 years showing no growth, disappearance or clear evidence of reduction in size of the pulmonary mass lesion.

\section{Statistical analysis}

The results of histological or cytological analysis, or proof of benign disease on radiological follow-up, were considered the gold standard and were correlated with the findings of the integrated PET-CT in order to obtain sensitivity, specificity, positive predictive value, negative predictive value and diagnostic accuracy of integrated PETCT. These were calculated by standard techniques, initially with an $\mathrm{SUV}_{\max }$ cutoff of 2.5. Thereafter a receiver operating characteristic (ROC) curve was obtained from the data set to determine an $\mathrm{SUV}_{\text {max }}$ cut-off that would provide the optimal sensitivity and specificity for our data set.

\section{Results}

\section{Study population and diagnoses}

Fifty patients fulfilled the inclusion criteria, but one was excluded owing to technical problems with acquisition of the PET study, leaving a study population of 49 patients (mean (standard deviation (SD)) age 60.1 (10.2) years; 29 males). The final diagnoses are summarised in Table 1. Lung cancer was confirmed in 30 patients, and two patients were found to have carcinoid tumours. Of the patients with malignant lesions, $21 / 32$ $(65.6 \%)$ were ultimately found to have resectable disease.

Table 1. Final aetiology in all cases $(N=49)$

\begin{tabular}{ll}
\hline Type & $\boldsymbol{n}(\%)$ \\
\hline Adenocarcinoma & $19(38.8)$ \\
Squamous cell carcinoma & $7(14.7)$ \\
Poorly differentiated carcinoma & $2(4.1)$ \\
Carcinoid & $2(4.1)$ \\
Active pulmonary TB & $8(16.3)$ \\
Other benign & $11(22.4)$ \\
* Other benign diagnoses included solitary fibrous tumour \\
$\begin{array}{l}(n=1), \text { old granulomas }(n=7), \text { bronchiectasis }(n=2) \text { and } \\
\text { Haemophilus influenzae infection }(n=1) .\end{array}$
\end{tabular}

Chest radiograph and CT findings

The average diameters of the lesions measured were $29 \mathrm{~mm}$ in the vertical axis and $30 \mathrm{~mm}$ in the horizontal axis on standard anteroposterior (AP) chest radiograph, and these were very similar to the CT measurements (Figs 1 and 2). Lesions ultimately proven to be malignant were most frequently observed in the right $(n=12)$ and left upper lobes $(n=8)$.

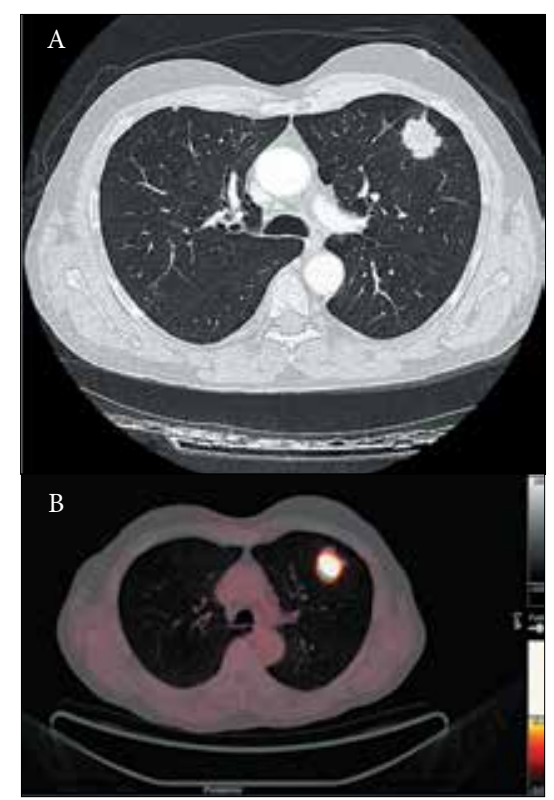

Fig. 1. A: An example of a mass lesion in the left upper lobe seen on CT scan. B: PET-CT showed high FDG uptake with an $S U V_{\text {max }}$ of 13.3. The final diagnosis in this case was squamous cell carcinoma.

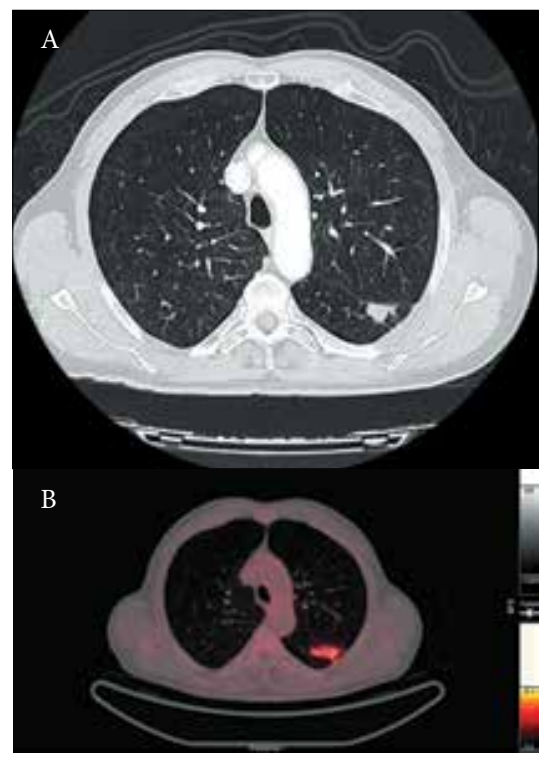

Fig. 2. A: CT scan from a patient who presented with a mass lesion in the apical segment of the left lower lobe. B: PET-CT confirmed an FDGavid lesion with an SUV $V_{\max }$ of 3.8. This patient was ultimately diagnosed with pulmonary TB. 
Table 2. Respective diagnostic accuracies of SUV $\mathrm{max}_{\max }$ cut-offs of $\mathbf{2 . 5}$ and 5.0

\begin{tabular}{lll}
\hline & SUV $_{\max } \mathbf{2 . 5}, \boldsymbol{\%}(\mathbf{9 5 \%} \mathbf{C I})$ & $\mathbf{S U V}_{\max } \mathbf{5 . 0}, \mathbf{\%}(\mathbf{9 5} \% \mathbf{C I})$ \\
\hline Sensitivity & $93.3(76.5-98.8)$ & $90.0(72.3-97.4)$ \\
Specificity & $36.8(17.2-61.4)$ & $78.9(53.9-93.0)$ \\
PPV & $70.0(53.3-82.9)$ & $87.1(69.2-95.8)$ \\
NPV & $77.8(40.2-96.1)$ & $83.3(57.7-95.6)$ \\
PPV = positive predictive value; $\mathrm{NPV}=$ negative predictive value. &
\end{tabular}

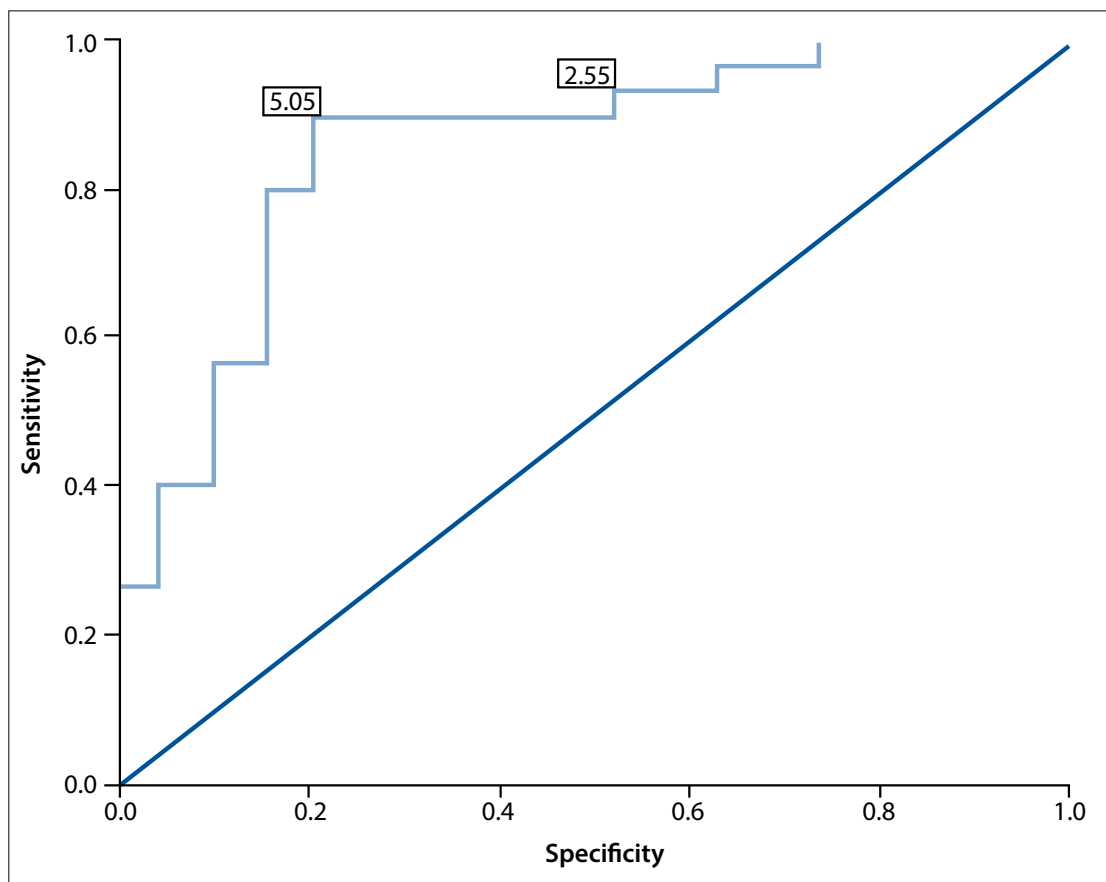

Fig. 3. The ROC curve, annotated with the $S U V_{\max }$ cut-off values of 2.5 and 5.0 (see text for details).

\section{PET-CT findings and SUV ${ }_{\max }$}

Malignant lesions had a higher mean (SD) $\mathrm{SUV}_{\text {max }}$ than lesions caused by TB (12.4 (6.7) v. $6.8(5.2), p=0.032)$. When the $\mathrm{SUV}_{\text {max }}$ cutoff of 2.5 was used, the sensitivity, specificity, positive predictive value, negative predictive value and diagnostic accuracy of integrated PET-CT were $93.3 \%, 36.8 \%, 70.0 \%, 77.8 \%$ and $71.4 \%$, respectively (Table 2 ).

A ROC curve was then obtained by comparing each $\mathrm{SUV}_{\max }$ with the gold standard (Fig. 3). A ROC curve with a statistically significant area under the curve was obtained (0.854 (95\% confidence interval $0.741-0.968)$, $p<0.01)$. From this curve we could obtain an $\mathrm{SUV}_{\max }$ that provided optimal sensitivity and specificity to our test. With a cut-off of 5.0 the sensitivity, specificity, positive predictive value, negative predictive value and diagnostic accuracy of integrated PET-CT for malignancy were $90.0 \%, 78.9 \%, 87.1 \%$, $83.3 \%$ and $86.7 \%$, respectively (Table 2). These analyses confirmed that an $\mathrm{SUV}_{\max }$ cut-off of 5.0 improved the specificity of integrated PET-CT for cancer in our setting from $36.8 \%$ to $78.9 \%$ and the diagnostic accuracy from $71.4 \%$ to $86.7 \%$, with only a small reduction in sensitivity from $93.3 \%$ to $90.0 \%$.

\section{Discussion}

We found PET-CT to have poor diagnostic accuracy in an area with a very high incidence of TB. Moreover, an $\mathrm{SUV}_{\max }$ threshold of 2.5 had a specificity of only $37 \%$. However,

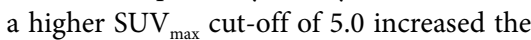
specificity to $79 \%$ and diagnostic accuracy from $71 \%$ to $87 \%$, with only a marginal reduction in sensitivity from $93 \%$ to $90 \%$.

International PET-CT data for the evaluation of SPNs show higher accuracy for cancer in settings where the TB prevalence is substantially lower. ${ }^{[4,9]} \mathrm{A}$ recent metaanalysis by Deppen et al. ${ }^{[4]}$ on the ability of PET-CT to diagnose lung cancer accurately in areas with a moderate burden of infectious lung diseases found significant heterogeneity for sensitivity and specificity across studies. The authors reported a pooled sensitivity of $99 \%$ and specificity of $75 \%$. Alarmingly, there was a $16 \%$ lower average adjusted specificity $(61 \%$ v. $77 \%)$ in regions with endemic infectious lung disease compared with non-endemic regions.

Gould et al ${ }^{[9]}$ previously showed that PET is an accurate, non-invasive method for diagnosing an SPN in a developed world setting, with an overall sensitivity of $96.8 \%$ and a specificity of $77.8 \%$. A retrospective study of 42 patients with SPNs in California found both an SUV max $_{\text {ax }}$ cut-off of 2.0 and the visual interpretation yielded similar accuracy, with a sensitivity and specificity of $97 \%$ and $85 \%$, respectively. ${ }^{[10]}$

In Brazil, with a TB incidence rate of $66 / 100000$ population, the sensitivity and specificity of PET-CT (using an SUV max $_{\text {max }}$ cutoff of 2.5 ) were found to be $92.9 \%$ and $72.2 \%$, respectively. ${ }^{[11]}$ Limited data exist for the evaluation of PET-CT in other TB-endemic areas. In a Chinese population this modality was found to have a sensitivity and specificity of $88.3 \%$ and $61.1 \%$, respectively. ${ }^{[12]}$ An SUV $_{\text {max }}$ cut-off of 3.96 (determined by a ROC curve analysis) improved the sensitivity to $73.3 \%$ and the specificity to $75.0 \%$ in this population. ${ }^{[12}$

Only three studies have evaluated nuclear scanning to assess pulmonary mass lesions in SA. ${ }^{[6,13,14]}$ Schuurmans et al. ${ }^{[13]}$ found a sensitivity of $92.3 \%$ and a specificity of $91.7 \%$ for the use of technetium 99m-methoxy isobutyl isonitrile as a marker for tumour uptake. Sathekge et al. ${ }^{[6]}$ performed a prospective study of 30 patients to assess the accuracy of dual time point PET-CT in the assessment of SPNs in Pretoria, SA. Their analysis showed similar findings to our study with a sensitivity of $85.7 \%$ and a specificity of $25 \%$ when using an SUV ${ }_{\text {max }}$ cut-off of 2.5 . When TB $(n=12)$ was excluded from their analysis, the sensitivity improved to $85.7 \%$ and the specificity to $100 \%$. The present study confirms the findings of Sathekge et al. ${ }^{[6]}$ regarding poor specificity in an area with high TB prevalence. Moreover, our data suggest that an $\mathrm{SUV}_{\max }$ of 5.0 may be a more appropriate cut-off value in an area with a high TB incidence, and additionally that values of 2.5 - 5.0 should be interpreted as indeterminate. In a recent study, Vorster et al. ${ }^{[14]}$ demonstrated that 68Ga-citrate PET/ CT has potential for the detection of both malignancy and TB, but it seemed incapable of providing a clear distinction between malignant and benign lung lesions in a setting with a high prevalence of granulomatous diseases such as TB.

Importantly, it should be emphasised that the interpretation of PET-CT does not rely solely on SUV values. Swensen et al., ${ }^{[15]}$ for example, developed a pretest probability model using age, smoking history, history 
of extrathoracic cancer more than 5 years before nodule detection, nodule diameter, upper lobe location and spiculated margin. They achieved an area under the ROC curve of 0.80 . Li et al ${ }^{[16]}$ developed a mathematical model for predicting malignancy using age, diameter, border calcification, spiculation, and family history of tumour. Their model generated a sensitivity of $94.5 \%$ and a specificity of $70.0 \%$. Tian et al. ${ }^{[17]}$ included FDG uptake as a variable in developing a prediction model by logistic regression analysis. They used gender, smoking history, age, diameter, spiculation and FDG uptake to estimate the probability of malignancy of SPNs. Using a cut-off value determined by ROC curve analysis, they achieved a sensitivity of $90.2 \%$ and a specificity of $87.6 \%$. PET-CT is therefore not a stand-alone investigation, but can contribute to the determination of malignant probability. Such a model could potentially be developed in a TB-endemic area.

A potential weakness of this study is the inclusion of a small proportion of patients initially assessed to have solitary pulmonary mass lesions or nodules on chest radiograph, who were later found to have more extensive disease on CT or PET-CT. Moreover, a limitation of increasing the $\mathrm{SUV}_{\max }$ to 5.0 is that a number of lung malignancies with low metabolic activity (such as adenocarcinoma in situ) may be falsely reported as negative, whereas some tuberculous lesions (which may demonstrate higher $\mathrm{SUV}_{\text {max }}$ values) may be interpreted as positive.

In conclusion, the reduced specificity for malignancy of PET-CT in TB-endemic areas can be significantly improved by using an optimised threshold for SUV $\mathrm{max}_{\max }$. PET-CT is a valuable adjunct in the assessment of patients with a pulmonary mass lesion on presentation, provided other clinical and radiological evidence is integrated in the assessment. Further research on integrating various SUVs with clinical and radiological prediction scores in settings with a high prevalence of TB is needed.

Acknowledgments. Tonya Esterhuizen of the Centre for Evidencebased Health Care, Stellenbosch University, performed the independent statistical analysis of study data.

\section{References}

1. Swensen S, Jett J, Payne W, et al. An integrated approach to evaluation of the solitary pulmonary nodule. Mayo Clin Proc 1990;65(2):173-186. [http://dx.doi.org:/10.1016/s0025-6196(12)65012-5]

2. Fischer B, Lassen U, Mortensen J, et al. Preoperative staging of lung cancer with combined PET-CT. N Engl J Med 2009;361(1):32-39. [http://dx.doi.org/10.1056/nejmoa0900043]

3. Shaw JA, Irusen EM, von Groote-Bidlingmaier F, et al. Integrated positron emission tomography/ computed tomography for evaluation of mediastinal lymph node staging of non-small-cell lung cancer in a tuberculosis-endemic area: A 5-year prospective observational study. S Afr Med J 2015;105(2):145150. [http://dx.doi.org/10.7196/SAMJ.8357]

4. Deppen SA, Blume JD, Kensinger CD, et al. Accuracy of FDG-PET to diagnose lung cancer in areas with Deppen SA, Blume JD, Kensinger CD, et al. Accuracy of FDG-PET to diagnose lung cancer in areas with
infectious lung disease. JAMA 2014;312(12):1227-1236. [http://dx.doi.org/10.1001/jama.2014.11488]

infectious lung disease. JAMA 2014;312(12):1227-1236. [http://dx.doi.org/10.1001/jama.2014.11488]
5. Chang J, Lee H, Goo J, et al. False positive and false negative FDG-PET scans in various thoracic 5. Chang J, Lee H, Goo J, et al. False positive and false negative FDG-PET scans in
diseases. Korean J Radiol 2006;7(1):57-69. [http://dx.doi.org/10.3348/kr.2006.7.1.57]

6. Sathekge M, Maes A, Pottel H, Stoltz A, van de Wiele C. Dual time-point FDG PET-CT for differentiating benign from malignant solitary pulmonary nodules in a TB endemic area. S Afr Med J 2010;100(9):598-601

7. World Health Organization. WHO Global Tuberculosis Report 2014. http://www.who.int/tb/ publications/global_report/en (accessed 15 February 2015).

8. Boellaard R, O’Doherty M, Weber W, et al. EANM procedure guidelines for tumour PET imaging: Version 1.0. Eur J Nucl Med Mol Imaging 2010;37(1):181-200. [http://dx.doi.org/10.1007/s00259-0091297-4]

9. Gould M, Maclean C, Kuschner W, Rydzak C, Owens D. Accuracy of positron emission tomography for diagnosis of pulmonary nodules and mass lesions: A meta-analysis. JAMA 2001;285(7):914-924. [http://dx.doi.org/10.1001/jama.285.7.914]

10. Kim S, Allen-Auerbach M, Goldin J, et al. Accuracy of PET/CT in characterization of solitary pulmonary lesions. J Nucl Med 2007;48(2):214-220.
pula

11. Martins Rde C, Almeida S, Siciliano A, et al. Value of [18F]-FDG-PET/CT as a predictor of cancer in solitary pulmonary nodule. J Bras Pneumol 2008;34(7):473-480. [http://dx.doi.org/10.1590/s1806-
inter in solitary pulmonary

12. Li Y, Su M, Li F, Kuang A, Tian R. The value of 18F-FDG-PET/CT in the differential diagnosis of solitary pulmonary nodules in areas with a high incidence of tuberculosis. Ann Nucl Med 2011;25(10):804811. [http://dx.doi.org/10.1007/s12149-011-0530-y]

13. Schuurmans M, Ellmann A, Bouma H, et al. Solitary pulmonary nodule evaluation with $99 \mathrm{mTc}$ methoxy isobutyl isonitrile in a tuberculosis-endemic area. Eur Respir J 2007;30(6):1090-1095. [http:// dx.doi.org/10.1183/09031936.00046107]

14. Vorster M, Maes A, Jacobs A, et al. Evaluating the possible role of $68 \mathrm{Ga}$-citrate PET/CT in the characterization of indeterminate lung lesions. Ann Nucl Med 2014;28(6):523-530. [http://dx.doi. org/10.1007/s12149-014-0842-9]

15. Swensen S, Silverstein M, Ilstrup D, Schleck C, Edell E. The probability of malignancy in solitary pulmonary nodules: Application to small radiologically indeterminate nodules. Arch Intern Med 1997;157(8):849-855. [http://dx.doi.org/10.1001/archinte.1997.00440290031002] 16. Li Y, Wang J. A mathematical model for predicting malignancy of solitary pulmonary nodules. World

17. Tian R, Su M, Tian Y, Li F, Kuang A. Development of a predicting model to estimate the probability of malignancy of solitary pulmonary nodules. Sichuan Da Xue Xue Bao Yi Xue Ban 2012;43:404-408. [http://dx.doi.org/10.1016/j.cllc.2011.06.005]

Accepted 5 November 2014. 